\title{
Biped Without Feet in Single Support: Stabilization of the Vertical Posture with Internal Torques
}

\author{
Formal'sky Alexander \\ Institute of Mechanics, Moscow Lomonosov State University \\ Russia \\ Aoustin Yannick \\ IRCCyN, École Centrale de Nantes, Université de Nantes \\ France
}

\section{Introduction}

We consider a two-link biped, a three-link biped, and a five-link planar biped without feet (with "point feet"). Their ankles are not actuated. The control torques are applied to the bipeds in the inter-link joints only. Then this family of bipeds is under actuated when only one leg tip touches the ground. It is difficult to control the walking of this kind of bipeds because they are statically unstable in single support. For example the vertical posture of these bipeds in single support is an unstable equilibrium state, as an equilibrium state of inverted pendulum. The operations of stabilization for the biped vertical posture, of balancing around this equilibrium posture, using only the inter-link torques, are also difficult. These problems are interesting from the point of view of dynamical stabilization of walking for bipeds with motions in saggital plane or (and) in frontal plane. They are also interesting from the biomechanical point of view. In the chapter, the problem of stabilization of the vertical posture for each mentioned above biped is studied. For each biped, a control law to stabilize the vertical posture is designed.

Among the mechanical systems, the under actuated systems, which have fewer controls than configuration variables, represent a great challenge for the control. An active field of research exists, due to the applications of under actuated systems such as aircrafts, satellites, I spacecrafts, flexible robots, inverted pendulums, legged robots. The under actuated systems कै are characterized by the under-actuation degree, which is the difference between the numbers of configuration variables and controls. The under-actuation degree for all our studied bipeds equals one in single support.

The control laws to stabilize the vertical posture are designed, using the biped linear models and their associated Jordan forms. The feedback is synthesized to suppress the unstable modes. The restrictions imposed to the torques are taken into account explicitly. Thus, feedback control laws with saturation are designed. It is important for an unstable system to maximize the basin of attraction. Using the Jordan form to design the control law, we can obtain a large basin of attraction of equilibrium state. 
With the aim to achieve fast walking gaits, some papers have been devoted to the study of walking mechanisms as the compass and the biped with point feet (see for example, (Spong et al., 2000); (Cambrini et al., 2001); (Canudas et al., 2002); (Aoustin \& Formal'sky, 2003); (Chevallereau et al., 2003); (Westervelt et al., 2003)). The study and control of walking and running gaits of these robots is a very interesting and simultaneously difficult problem. The challenge of the control law is to "suppress" the instability of these statically unstable and under actuated objects and also to reduce the time of transient oscillations. In (Cambrini et al., 2001), it is shown that it is possible to track in single support stable trajectories with internal stability by a suitable choice of outputs for a two-link robot and for a five-link robot. The authors in (Canudas et al., 2002); (Aoustin \& Formal'sky, 2003); (Chevallereau et al., 2003); (Chevallereau et al., 2004) realize orbital stabilization for a five-link biped, also in the single support phase. For the family of bipeds with internal torques, it is possible dynamically to stabilize their specific walking gaits. For example, in (Grizzle et al., 2001) authors, using the Poincaré map, proved that it is possible to ensure the dynamical stability of a three-link biped under a control law being finite time convergent. In (Aoustin \& Formal'sky, 2003), the convergence to a nominal cyclic motion is improved, by changing the step length or the trunk orientation.

Usually the limits imposed on the torques are not taken into account explicitly. For the problem of posture stabilization we propose a strategy of control with restricted torques. The control law is defined such that the torques adjust only the unstable modes of the biped. The numerical investigations of nonlinear models of the mentioned bipeds with the designed control laws are presented. The efficiency of the designed controls is shown.

In our opinion, the described approach here is useful for unstable systems of different kind. It is possible to apply this approach for the stabilization of inverted pendulums, for stabilization of monocycle (Beznos et al., 2003); (Aoustin et al., 2005); (Aoustin et al., 2006); (Formal'sky, 2006); (Martynenko \& Formal'sky, 2005).

The organization of this chapter is the following. Section 2 is devoted to the model of the biped. It contains also the data of the physical parameters of the five-link biped. The linear model of the biped motion around the vertical posture is presented in Section 3. The statement of the problem is defined in Section 4. The control law for the two-link biped is designed in Section 5. The control laws for the three-link and five-link bipeds are developed in Sections 6 and 7 respectively. Section 8 presents our conclusion and perspectives.

\section{Model Description of the Planar Biped}

\subsection{The dynamic model}

We consider an under actuated planar biped with $n$ degrees of freedom and $n-1$ actuators. Thus, the under-actuation degree for our biped equals one in single support. The generalized forces (torques) are only due to the actuators in the inter-link joints. The dynamic model of the biped single support motion is given by the following Lagrange matrix equation:

$$
\mathrm{D}(\mathrm{q}) \ddot{\mathrm{q}}+\mathrm{C}(\mathrm{q}, \dot{\mathrm{q}})+\mathrm{F} \dot{\mathrm{q}}+\mathrm{G}(\mathrm{q})=\mathrm{B} \Gamma
$$

Here, $\mathrm{q}$ is the $\mathrm{n} \times 1$ configuration vector. Its coordinates are the absolute angle between the trunk and the vertical axis, and the $n-1$ actuated inter-link angles. $D(q)$ is the $n \times n$ inertia positive definite matrix, $C(q, \dot{q})$ is the $n \times 1$ column of Coriolis and centrifugal forces. The matrix $\mathrm{D}(\mathrm{q})$ depends on the $\mathrm{n}-1$ inter-link angles only. We assume that at each actuated joint there is a viscous friction. Let the friction coefficient $\mathrm{f}$ be identical in 
all actuated joints, $F=\left(\begin{array}{ll}0_{1 \times 1} & 0_{1 \times(n-1)} \\ 0_{(n-1) \times 1} & \mathrm{fI}_{n-1}\end{array}\right)$, where $\mathrm{I}_{\mathrm{n}-1}$ is a $(\mathrm{n}-1) \times(\mathrm{n}-1)$ unit matrix. $\mathrm{G}(\mathrm{q})$ is the $n \times 1$ vector of the torques due to gravity. $B$ is a constant $n \times(n-1)$ matrix, $\Gamma$ is the $(n-1) \times 1$ vector of the torques, applied in the knee and hip joints. The diagrams of the two-link biped $(n=2)$, the three-link biped $(n=3)$, and the five-link biped $(n=5)$ are presented in Figure 1. The model (1) is computed considering that the contact between the tip of the stance leg and the ground is an undriven pivot. But in reality there is a unilateral constraint between the ground and the stance leg tip: the ground cannot prevent the stance leg from taking off. We assume there is no take off and no sliding. Thus, it is necessary to check the ground reaction in the stance leg tip. Its vertical component $\mathrm{R}_{\mathrm{y}}$ must be directed upwards. We introduce the following equations applying Newton's second law for the center of mass of the biped to determine the ground reaction $R\left(R_{x}, R_{y}\right)$,

$$
\begin{aligned}
& R_{x}=M \ddot{x}_{c} \\
& R_{y}=M \ddot{y}_{c}+M g
\end{aligned}
$$

Here, $\mathrm{M}$ is the total mass of the biped, $\mathrm{x}_{\mathrm{c}}$ and $\mathrm{y}_{\mathrm{c}}$ are the coordinates of the mass center of the biped. To check if the ground reaction is located in the friction cone, we have to calculate the ratio $R_{x} / R_{y}$.
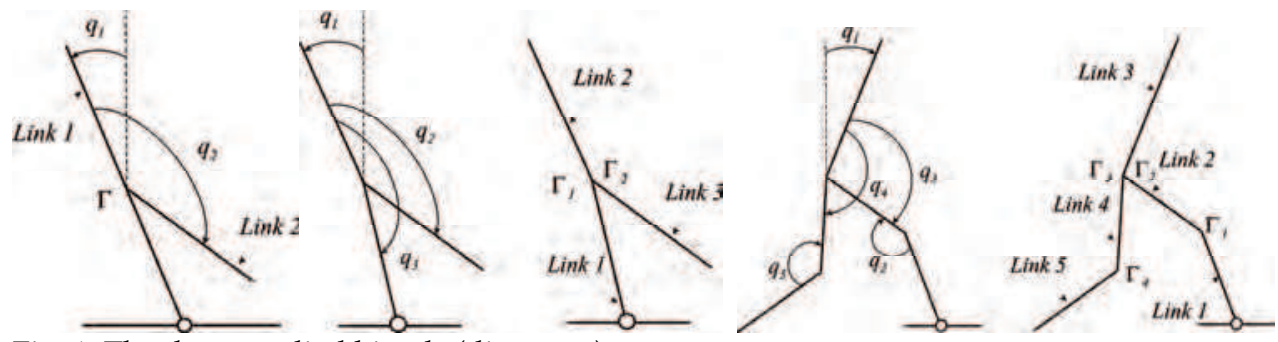

Fig. 1. The three studied bipeds (diagrams).

\subsection{The physical parameters of dynamic model}

For the numerical experiments we use the physical parameters of the five-link biped prototype "Rabbit" (Chevallereau et al., 2003).

We assume that both legs are identical (see Figure 1, two last diagrams). The masses of the shins are: $\mathrm{m}_{1}=\mathrm{m}_{5}=3.2 \mathrm{~kg}$; the masses of the thighs are: $\mathrm{m}_{2}=\mathrm{m}_{4}=6.8 \mathrm{~kg}$; the mass of the trunk is: $\mathrm{m}_{3}=16.5 \mathrm{~kg}$. The lengths of the shins and the thighs are identical: $1_{1}=l_{2}=l_{3}=l_{4}=1_{5}=0.4 \mathrm{~m}$; the length of the trunk is: $1_{3}=0.625 \mathrm{~m}$. The height of the biped equals $1.425 \mathrm{~m}$, the total mass $\mathrm{M}$ equals $36.5 \mathrm{~kg}$.

The distances between the mass center of each link and the corresponding joint are the following: $\mathrm{s}_{1}=\mathrm{s}_{5}=0.127 \mathrm{~m}, \mathrm{~s}_{2}=\mathrm{s}_{4}=0.163 \mathrm{~m}$ and $\mathrm{s}_{3}=0.2 \mathrm{~m}$.

The inertia moments around the mass center of each link are: $I_{1}=I_{5}=0.0484 \mathrm{~kg} \cdot \mathrm{m}^{2}$, $\mathrm{I}_{2}=\mathrm{I}_{4}=0.0693 \mathrm{~kg} \cdot \mathrm{m}^{2}$ and $\mathrm{I}_{3}=1.9412 \mathrm{~kg} \cdot \mathrm{m}^{2}$. The inertia of the rotor for each DC motor is 
$\mathrm{I}=3.32 \cdot 10^{-4} \mathrm{~kg} \cdot \mathrm{m}^{2}$.

All the gear ratios are identical and equal 50. The maximum value $U$ of the torques equals $150 \mathrm{~N} \cdot \mathrm{m}$.

Using these values we have also calculated the corresponding values for the two-link and three-link bipeds. In Section 5, we calculate the parameters of the two-link biped. For example, the mass of the first link of the two-link biped (see Figure 1, first diagram) equals $\mu_{1}=m_{3}+m_{4}+m_{5}$. The mass of its second link (of the leg) equals $\mu_{2}=m_{2}+m_{1}+m_{4}+m_{5}$. The length of the first link equals $1_{3}+1_{4}+1_{5}$, the length of its second link (of the leg) equals $1=l_{2}+l_{1}=l_{4}+l_{5}$. The distance $r_{1}$ between the unique inter-link joint and the center of mass of the first link equals

$$
\mathrm{r}_{1}=\frac{-\mathrm{m}_{3} \mathrm{~s}_{3}+\mathrm{m}_{4} \mathrm{~s}_{4}+\mathrm{m}_{5}\left(\mathrm{l}_{4}+\mathrm{s}_{5}\right)}{\mathrm{m}_{3}+\mathrm{m}_{4}+\mathrm{m}_{5}}
$$

The distance $r_{2}$ between the inter-link joint and the center of mass of the second link equals

$$
\mathrm{r}_{2}=\frac{\mathrm{m}_{2} \mathrm{~s}_{2}+\mathrm{m}_{1}\left(\mathrm{l}_{2}+\mathrm{s}_{1}\right)}{\mathrm{m}_{2}+\mathrm{m}_{1}}=\frac{\mathrm{m}_{4} \mathrm{~s}_{4}+\mathrm{m}_{5}\left(\mathrm{l}_{4}+\mathrm{s}_{5}\right)}{\mathrm{m}_{4}+\mathrm{m}_{5}}
$$

It follows from (3) and (4) that

$$
1-r_{1}>0,1-r_{2}>0, r_{2}-r_{1}>0
$$

In Section 6, we calculate the parameters of the three-link biped. The mass of each leg (see Figure 1 , second and third diagrams) equals $\mu_{3}=\mathrm{m}_{2}+\mathrm{m}_{1}=\mathrm{m}_{4}+\mathrm{m}_{5}$. The mass of its trunk is $\mathrm{m}_{3}$. The length of each leg equals $1=1_{2}+1_{1}=1_{4}+1_{5}$, the length of the trunk is $1_{3}$. The distance between the inter-link joint and the center of mass of each leg equals $r_{2}$ and is defined by the formula (4). The distance between the inter-link joint and the mass center of the trunk equals $s_{3}$.

\section{Linear Model of the Planar Biped}

In this section, we present the matrix equation (1) linearized around the vertical posture of the biped, the state form of this linear model and its Jordan form. This Jordan form will be useful to define the control laws in the next sections.

Let $\mathrm{q}_{\mathrm{e}}$ denote the configuration vector of the biped in the vertical posture. This vertical posture is an equilibrium position. This equilibrium point is $\mathrm{q}_{\mathrm{e}}=(0, \pi)^{\mathrm{T}}$ for the two-link biped, $q_{e}=(0, \pi, \pi)^{T}$ for the three-link biped, and $q_{e}=(0, \pi, \pi, \pi, \pi)^{T}$ for the five-link biped. The linear model is defined by the variation vector $v=q-q_{e}$ of the configuration vector $q$ around the vertical equilibrium posture $q_{e}$,

$$
\mathrm{D}_{l} \ddot{\mathrm{v}}+\mathrm{F} \dot{\mathrm{v}}+\mathrm{G}_{l} v=\mathrm{B} \Gamma
$$

Here, $D_{l}$ is the inertia matrix for the configuration vector $\mathrm{q}_{\mathrm{e}}: \mathrm{D}_{l}=\mathrm{D}\left(\mathrm{q}_{\mathrm{e}}\right) \cdot \mathrm{G}_{l}$ is the Jacobian of the matrix $G(q)$ computed at the equilibrium point $q_{e}$. We will consider the following 
constraint imposed on each torque:

$$
\left|\Gamma_{\mathrm{i}}\right| \leq \mathrm{U},(\mathrm{i}=1, \ldots, \mathrm{n}-1), \mathrm{U}=\mathrm{const}
$$

We deduce from (6) the state model with $x=(v, \dot{v})^{T}$ :

$$
\dot{\mathrm{x}}=\mathrm{Ax}+\mathrm{b} \Gamma
$$

Here,

$$
\mathrm{A}=\left(\begin{array}{cc}
0_{\mathrm{n} \times \mathrm{n}} & \mathrm{I}_{\mathrm{n} \times \mathrm{n}} \\
-\mathrm{D}_{l}^{-1} \mathrm{G}_{l} & -\mathrm{D}_{l}^{-1} \mathrm{~F}
\end{array}\right), \mathrm{b}=\left(\begin{array}{c}
0_{\mathrm{n} \times(\mathrm{n}-1)} \\
\mathrm{D}_{l}^{-1} \mathrm{~B}
\end{array}\right)
$$

Introducing a nondegenerate linear transformation $x=S y$, with a constant matrix $S$, we are able to obtain the well-known Jordan form (Ogata, 1990) of the matrix equation (8):

$$
\dot{\mathrm{y}}=\Lambda \mathrm{y}+\mathrm{d} \Gamma
$$

where

$$
\mathrm{L}=\mathrm{S}^{-1} \mathrm{AS}=\left(\begin{array}{cccc}
\mathrm{l}_{1} & & & 0 \\
& \cdot & & \\
& & \cdot & \\
& & \cdot & \\
0 & & & 1_{2 \mathrm{n}}
\end{array}\right), \mathrm{d}=\mathrm{S}^{-1} \mathrm{~b}
$$

Here, $\lambda_{1}, \ldots, \lambda_{2 n}$ are the eigenvalues of the matrix $A, d$ is $2 n \times(n-1)$ matrix. Let us consider that the positive eigenvalues have the smaller subscript. For the two-link biped we will obtain $\lambda_{1}>0, \operatorname{Re} \lambda_{\mathrm{i}}<0 \quad(\mathrm{i}=2,3,4)$, for the three-link biped $\lambda_{1}>0, \lambda_{2}>0, \operatorname{Re} \lambda_{\mathrm{i}}<0$ $(i=3-6)$, and for the five-link biped $\lambda_{i}>0 \quad(i=1,2,3), \operatorname{Re} \lambda_{j}<0 \quad(j=4-10)$.

\section{Problem Statement}

Let $x=0$ (here 0 is a $2 n \times 1$ zero-column) be the desired equilibrium state of the system (8). Let us design the feedback control to stabilize this equilibrium state $x=0$ under the constraint (7). In other words, we want to design an admissible (satisfying the inequality (7)) feedback control to ensure the asymptotic stability of the desired state $x=0$.

Let $\mathrm{W}$ be the set of the vector-functions $\Gamma(\mathrm{t})$ such that their components $\Gamma_{\mathrm{i}}(\mathrm{t})$ $(\mathrm{i}=1, \ldots, \mathrm{n}-1)$ are piecewise continuous functions of time, satisfying the inequalities (7). Let $Q$ be the set of the initial states $x(0)$ from which the origin $x=0$ can be reached, using an admissible control vector-function. Thus, the system (8) can reach the origin $x=0$ with the control $\Gamma(t) \in W$, only when starting from the initial states $x(0) \in Q$. The set $Q$ is called controllability domain. If the matrix $\mathrm{A}$ has eigenvalues with positive real parts and the control torques $\Gamma_{\mathrm{i}}(\mathrm{t})(\mathrm{i}=1, \ldots, \mathrm{n}-1)$ are limited, then the controllability domain $\mathrm{Q}$ is an open subset of the phase space $X$ for the system (8) (see (Formal'sky, 1974)). 
For any admissible feedback control $\Gamma=\Gamma(\mathrm{x}) \quad\left(\left|\Gamma_{\mathrm{i}}(\mathrm{x})\right| \leq \mathrm{U}, \mathrm{i}=1, \ldots, \mathrm{n}-1\right)$ the corresponding basin of attraction $\mathrm{V}$ belongs to the controllability domain: $\mathrm{V} \subset \mathrm{Q}$. Here as usual, the basin of attraction is the set of initial states $x(0)$ from which the system (8), with the feedback control $\Gamma=\Gamma(x)$ asymptotically tends to the origin point $x=0$ as $t \rightarrow \infty$. Some eigenvalues of the matrix A of the system (8), (10) are located in the left-half complex plane. The other eigenvalues of matrix $\mathrm{A}$ are in the right-half complex plane. We will design a control law, which "transfers" these last eigenvalues to the left-half complex plane.

The structure and the properties of this control law depend on the studied biped and its number of D.O.F. We will detail now these different cases.

\section{Two-Link Biped $(\mathbf{n}=\mathbf{2})$}

Here, we design a control law for a unique inter-link torque to stabilize the two-link planar biped, with a basin of attraction as large as possible.

\subsection{Control design for the two-link biped}

If the coefficient of the viscous friction $\mathrm{f}$ in the unique inter-link joint of the two-link biped is equal to zero, then the characteristic equation of the system (8), (10) with $\Gamma=0$ is biquadratic one

$$
\mathrm{a}_{0} \lambda^{4}+\mathrm{a}_{1} \lambda^{2}+\mathrm{a}_{2}=0
$$

with

$$
\mathrm{a}_{0}=\operatorname{det} \mathrm{D}_{l}, \mathrm{a}_{2}=\operatorname{det} \mathrm{G}_{l}=-\mu_{2} \mathrm{~g}^{2} \mathrm{r}_{2}\left[\mu_{1}\left(1-\mathrm{r}_{1}\right)+\mu_{2} \mathrm{l}\right] .
$$

The leading coefficient $a_{0}$ of the equation (12) is positive because it is the determinant of the positive definite matrix $D_{l}$. The free term $a_{2}$ of the equation (12) is negative because the difference $1-r_{2}$ is positive (see inequalities (5)). Therefore, the spectrum of the matrix $A$ is symmetric with respect to the imaginary axis and it is naturally because with $f=0$ the system (8) is conservative. This property is true in the general case, i.e., for a biped with $n$ links. In the case $n=2$ (under condition $f=0$ ), the matrix $A$ has two real eigenvalues (positive and negative), and two pure imaginary conjugate eigenvalues. If $f \neq 0$, then the matrix A for $\mathrm{n}=2$ has one real positive eigenvalue, and three eigenvalues in the left-half complex plane. Let $\lambda_{1}$ be the real positive eigenvalue, and let us consider the first scalar differential equation of the system (10) corresponding to this eigenvalue $\lambda_{1}$,

$$
\dot{\mathrm{y}}_{1}=\lambda_{1} \mathrm{y}_{1}+\mathrm{d}_{1} \Gamma
$$

The determinant of the controllability matrix (Kalman, 1969) for the linear model (8) is not null, if and only if:

$$
r_{1}\left(1-r_{1}\right)\left[J_{2}+\mu_{2} r_{2}\left(r_{2}-r_{1}\right)\right]+\mu_{2}\left(r_{2} J_{1}+1 J_{2}\right) \neq 0
$$

Here $J_{1}$ and $J_{2}$ are the inertia moments of the first and second links respectively around their mass centers. According to inequalities (5), the differences $1-r_{1}$ and $r_{2}-r_{1}$ are positive. Therefore, the inequality (14) is satisfied and then (8) is a Kalman controllable system, then the scalar inequality $d_{1} \neq 0$ is verified. The controllability region $Q$ of the equation (13) and 
consequently of the system (10) is described by the following inequality (Formal'sky, 1974),

$$
\left|\mathrm{y}_{1}\right|<\left|\mathrm{d}_{1}\right| \frac{\mathrm{U}}{\lambda_{1}}
$$

Thus, the controllability domain (15) is a strip in the space $\mathrm{Y}$ or $\mathrm{X}$.

We can "suppress" the instability of coordinate $y_{1}$ by a linear feedback control,

$$
\Gamma=\gamma y_{1}
$$

with the condition,

$$
\lambda_{1}+\mathrm{d}_{1} \gamma_{1}<0
$$

For the system (8), (9) with $\mathrm{n}=2$ under the feedback control (16) only one eigenvalue $\lambda_{1}$ (positive one) of the matrix $A$ is replaced by a negative value $\lambda_{1}+d_{1} \gamma_{1}$. The eigenvalues $\lambda_{2}$, $\lambda_{3}, \lambda_{4}$ do not change.

If we take into account the constraints (7), we obtain from (16) a linear feedback control with saturation,

$$
\Gamma=\Gamma\left(\mathrm{y}_{1}\right)=\left\{\begin{array}{ccc}
\mathrm{U}, & \text { if } & \gamma \mathrm{y}_{1} \geq \mathrm{U} \\
\gamma \mathrm{y}_{1}, & \text { if } & \left|\gamma \mathrm{y}_{1}\right| \leq \mathrm{U} \\
-\mathrm{U}, & \text { if } & \gamma \mathrm{y}_{1} \leq-\mathrm{U}
\end{array}\right.
$$

It is possible to see that if $\left|\mathrm{y}_{1}\right|<\left|\mathrm{d}_{1}\right| \mathrm{U} / \lambda_{1}$ (see inequality (15)), then under condition (17) the right part of the equation (13) with the control (18) is negative when $\mathrm{y}_{1}>0$ and positive when $\mathrm{y}_{1}<0$. Consequently, if $\left|\mathrm{y}_{1}(0)\right|<\left|\mathrm{d}_{1}\right| \mathrm{U} / \lambda_{1}$, then the solution $\mathrm{y}_{1}(\mathrm{t})$ of system (13), (18) tends to 0 as $t \rightarrow \infty$. But if $y_{1}(t) \rightarrow 0$, then according to the expression (18) $\Gamma(t) \rightarrow 0$ as $t \rightarrow \infty$. Therefore, the solutions $y_{i}(t) \quad(i=2,3,4$,) of the second, third and fourth equations of the system (10) with any initial conditions $\mathrm{y}_{\mathrm{i}}(0) \quad(\mathrm{i}=2,3,4$,) converge to zero as $\mathrm{t} \rightarrow \infty$ because $\operatorname{Re} \lambda_{i}<0$ for $i=2,3,4$. Thus, under the control (18) and with the inequality (17), the basin of attraction $\mathrm{V}$ coincides with the controllability domain Q, (Formal'sky, 1974); (Grishin et al., 2002): V = Q. So, the basin of attraction V for the system (8), (18) is as large as possible and it is described by the inequality (15).

Note that the variable $\mathrm{y}_{1}$ depends on the original variables from vector $\mathrm{x}$ according to the transformation $\mathrm{x}=\mathrm{Sy}$ or $\mathrm{y}=\mathrm{S}^{-1} \mathrm{x}$. Due to this, formula (18) defines the control feedback, which depends on the vector $x$ of the original variables.

According to Lyapounov's theorem, see (Slotine \& Li, 1991), the equilibrium $q=q_{e}$ of the nonlinear system (1) is asymptotically stable under the control (18) with some basin of attraction.

\subsection{Numerical results for the two-link biped}

We use the parameters defined in Section 2, the expressions (3), (4) and the friction coefficient $\mathrm{f}=6.0 \mathrm{~N} \cdot \mathrm{m} / \mathrm{s}$ to compute the parameters of the dynamic model for the two-link biped. The eigenvalues of the matrix $\mathrm{A}$ for the used parameters are the following:

$$
\lambda_{1}=3.378, \lambda_{2}=-3.406, \lambda_{3,4}=-1.510 \pm 3.584 \mathrm{i} .
$$

In simulation the control law (18) is applied to the nonlinear model (1) for the two-link biped. For the initial configuration $\mathrm{q}(0)=\left[1.94^{\circ} ; 180^{\circ}\right]$ the graphs of the variables $\mathrm{q}_{1}(\mathrm{t})$ and $\mathrm{q}_{2}(\mathrm{t})$ as functions 
of time are shown in Figure 2. At the end the biped is steered to the vertical posture. All the potential of the actuator is applied at the initial time, as shown in Figure 3. The basin of attraction for the nonlinear system depends on the feedback gain $\gamma$, which is chosen as -10000 in our numerical experiment. For the linear case, the relation (15) gives the maximum possible deviation of the two-link biped from the vertical axis $7.32^{\circ}$. But for the nonlinear model the maximum possible deviation is close to $1.94^{\circ}$. For each numerical experiment, we check the ground reaction in the stance leg tip to be sure that its vertical component is directed upwards. Figure 4 shows that during the stabilization process of the two-link biped, the vertical component of the ground reaction is always positive. Initially this component of the ground reaction is "large" and equals the weight of the biped at the end of the process.

We tested our strategy for different values of the actuator power. In fact, we considered different values $U$ for the maximum torque $\Gamma$. Figure 5 shows that the allowable deviation of the biped from the vertical posture increases nonlinearly with increasing of the torque maximum U. And this allowable deviation is limited. For the linear model this dependence is linear function of the torque maximum $U$ (see inequality (15)).
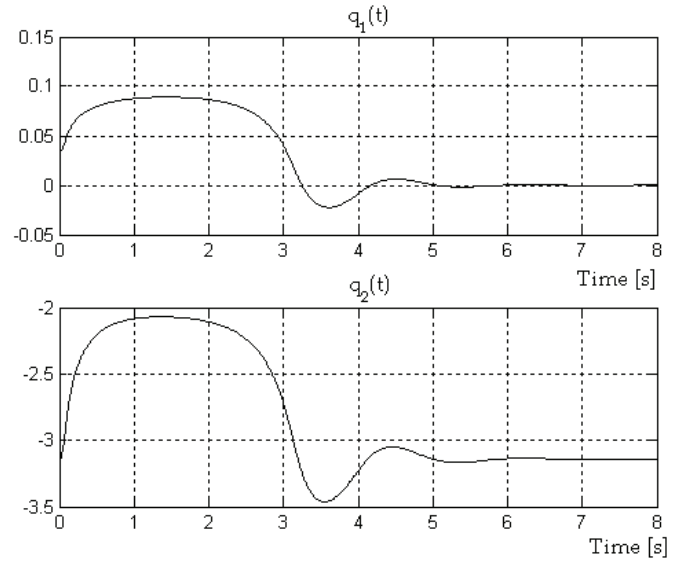

Fig. 2. Stabilization of the two-link biped in vertical posture, $\mathrm{q}_{1}(\mathrm{t}) \rightarrow 0$ and $\mathrm{q}_{2}(\mathrm{t}) \rightarrow-\pi$ as $\mathrm{t} \rightarrow \infty$.

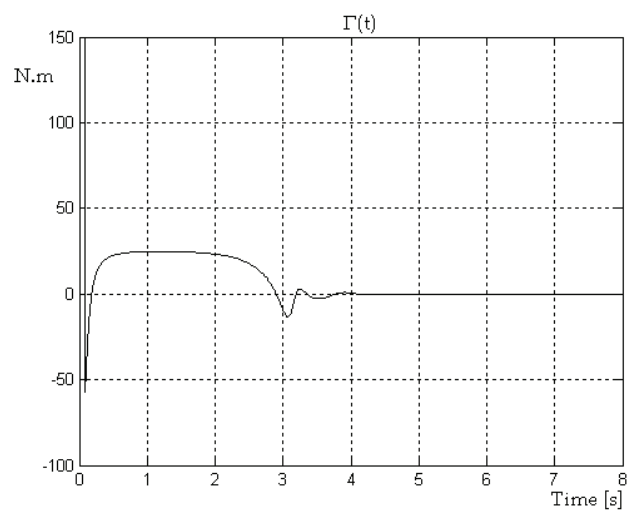

Fig. 3. Stabilization of the two-link biped in vertical posture, torque in the inter-link joint. 


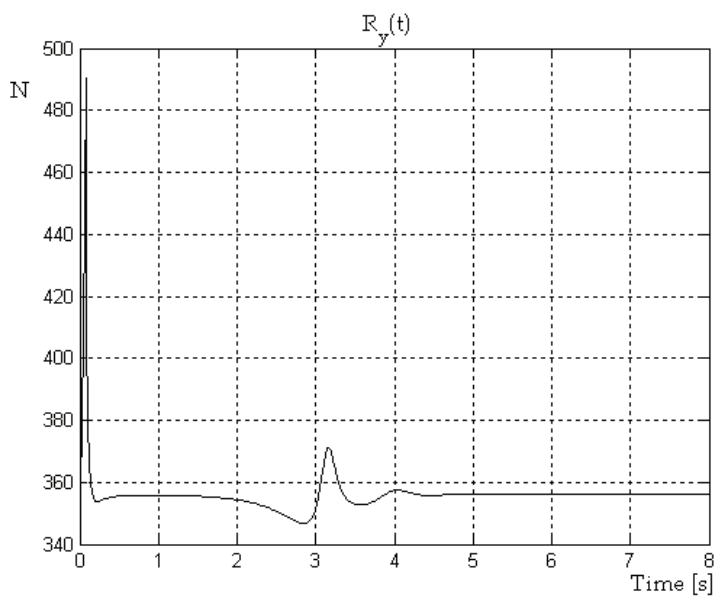

Fig. 4. Stabilization of the two-link biped in vertical posture, vertical component of the ground reaction in the stance leg tip.

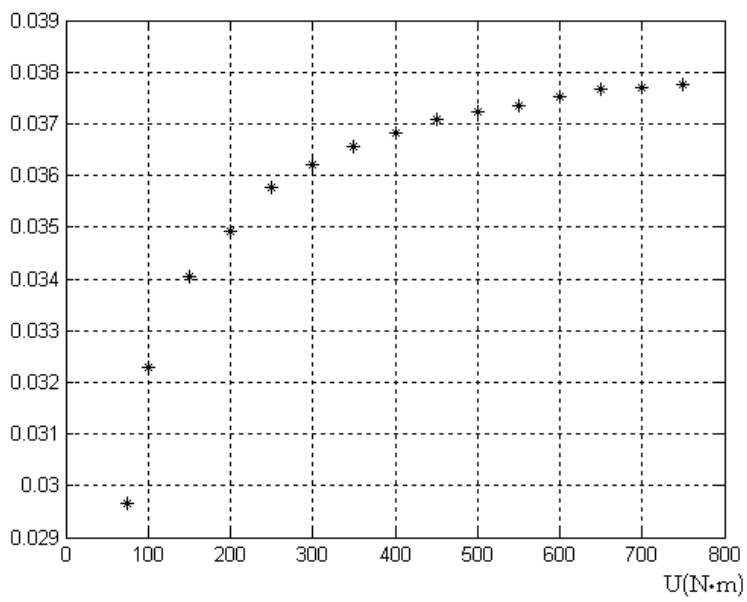

Fig. 5. Stabilization of the two-link biped, maximum allowable deviation of the biped from the vertical posture as a function of the maximum amplitude of the actuator torque.

\section{Three-Link Biped $(n=3)$}

In this paragraph, we design a control law for the two inter-link torques to stabilize the vertical posture of the three-link planar biped in single support.

\subsection{Control design for the three-link biped}

If the coefficient of the viscous friction $f=0$, the characteristic equation of the matrix $A$ of the linear model (8), (9) of the biped is bicubic one

$$
a_{0} p^{3}+a_{1} p^{2}+a_{2} p+a_{3}=0, p=\lambda^{2}
$$


with

$$
\mathrm{a}_{0}=\operatorname{det} \mathrm{D}_{l}, \quad \mathrm{a}_{3}=\operatorname{det} \mathrm{G}_{l}=\mu_{2} \mathrm{~m}_{3} \mathrm{~g}^{3} \mathrm{r}_{2} \mathrm{~s}_{3}\left[\mu_{2}\left(1-\mathrm{r}_{2}\right)+1\left(\mu_{2}+\mathrm{m}_{3}\right)\right]
$$

The system (8), (9) describes small oscillations of the biped. According to Sylvester's theorem (Chetaev, 1962), (Chetaev, 1989) the roots $\mathrm{p}_{1}, \mathrm{p}_{2}, \mathrm{p}_{3}$ of its characteristic equation (19) are real values. Thus, the spectrum of the matrix $A$ with $f=0$ is symmetric with respect to the imaginary axis. The leading coefficient $\mathrm{a}_{0}$ of the equation (19) is positive because it is the determinant of the positive definite matrix $D_{l}$. The free term $a_{3}$ of the equation (19) is positive because the difference $1-r_{2}$ is positive (see the inequalities (5)). Thus, according to Viet formula $p_{1} p_{2} p_{3}=-\frac{a_{3}}{a_{0}}$, the product $p_{1} p_{2} p_{3}$ is negative. This means that one or three roots are negative. In the first case, two roots are positive, in the second case, there are no positive roots. But at least one root for the studied unstable system must be positive. Consequently, two roots, for example $\mathrm{p}_{1}$ and $\mathrm{p}_{2}$, are positive and one is negative. Then spectrum of the matrix A contains two real positive eigenvalues, two real negative eigenvalues and two pure imaginary conjugate eigenvalues. If $f \neq 0$, then spectrum of the matrix A contains two values in the right-half complex plane, and four values in the left-half complex plane.

Let $\lambda_{i},(i=1,2)$ be real positive eigenvalues of the matrix $A$ (with $f \neq 0$ ), and let us consider the first two lines of the matrix equation (10) corresponding to these eigenvalues,

$$
\begin{aligned}
& \dot{\mathrm{y}}_{1}=\lambda_{1} \mathrm{y}_{1}+\mathrm{d}_{11} \Gamma_{1}+\mathrm{d}_{12} \Gamma_{2} \\
& \dot{\mathrm{y}}_{2}=\lambda_{2} \mathrm{y}_{2}+\mathrm{d}_{21} \Gamma_{1}+\mathrm{d}_{22} \Gamma_{2}
\end{aligned}
$$

The torques are chosen such that, the instability of both coordinates $y_{i},(i=1,2)$ is suppressed by choosing the feedback as,

$$
\begin{aligned}
& d_{11} \Gamma_{1}+d_{12} \Gamma_{2}=\gamma_{1} y_{1} \\
& d_{21} \Gamma_{1}+d_{22} \Gamma_{2}=\gamma_{2} y_{2}
\end{aligned}
$$

with both conditions,

$$
\lambda_{\mathrm{i}}+\gamma_{\mathrm{i}}<0 \quad(\mathrm{i}=1,2)
$$

Calculating both torques $\Gamma_{1}$ and $\Gamma_{2}$ from the algebraic equations (21), we obtain

$$
\begin{aligned}
& \Gamma_{1}=\frac{\gamma_{1} \mathrm{~d}_{22} \mathrm{y}_{1}-\gamma_{2} \mathrm{~d}_{12} \mathrm{y}_{2}}{\mathrm{~d}_{11} \mathrm{~d}_{22}-\mathrm{d}_{12} \mathrm{~d}_{21}}=\Gamma_{1}^{0}\left(\mathrm{y}_{1}, \mathrm{y}_{2}\right) \\
& \Gamma_{2}=\frac{\gamma_{2} \mathrm{~d}_{11} \mathrm{y}_{2}-\gamma_{1} \mathrm{~d}_{21} \mathrm{y}_{1}}{\mathrm{~d}_{11} \mathrm{~d}_{22}-\mathrm{d}_{12} \mathrm{~d}_{21}}=\Gamma_{2}^{0}\left(\mathrm{y}_{1}, \mathrm{y}_{2}\right)
\end{aligned}
$$

We assume here that the denominator in the expressions (23) is not zero.

For the system (10) with $n=3$ under the feedback control (23) two positive eigenvalues $\lambda_{1}$ and $\lambda_{2}$ of the matrix $A$ are replaced by negative values $\lambda_{1}+\gamma_{1}$ and $\lambda_{2}+\gamma_{2}$ respectively. All another eigenvalues do not change. 
Taking into account the constrains (7), the applied torques are,

$$
\Gamma_{\mathrm{i}}=\Gamma_{\mathrm{i}}\left(\mathrm{y}_{1}, \mathrm{y}_{2}\right)=\left\{\begin{array}{cccc}
\mathrm{U}, & \text { if } & \Gamma_{\mathrm{i}}^{0}\left(\mathrm{y}_{1}, \mathrm{y}_{2}\right) \geq \mathrm{U} \\
\Gamma_{\mathrm{i}}^{0}\left(\mathrm{y}_{1}, \mathrm{y}_{2}\right), & \text { if } & \mid \Gamma_{\mathrm{i}}^{0}\left(\mathrm{y}_{1}, \mathrm{y}_{2}\right) \leq \mathrm{U} \quad(\mathrm{i}=1,2) \\
-\mathrm{U}, & \text { if } & \Gamma_{\mathrm{i}}^{0}\left(\mathrm{y}_{1}, \mathrm{y}_{2}\right) \leq-\mathrm{U}
\end{array}\right.
$$

Under the control law (24), the equilibrium point $x=0$ is asymptotically stable for the system (8), (9) in some basin of attraction $\mathrm{V} \subset \mathrm{Q}$.

Using Lyapounov's theorem (Slotine, 1991), we can prove that the equilibrium point $q=q_{e}$ of the nonlinear system (1), (24) is asymptotically stable as well.

\subsection{Numerical results for the three-link biped}

We use the parameters defined in Section 2 and the friction coefficient $f=6.0 \mathrm{~N} \cdot \mathrm{m} / \mathrm{s}$ to compute the parameters of the dynamic model for the three-link biped. The eigenvalues of the matrix $\mathrm{A}$ for the used parameters are the following

$$
\lambda_{1}=3.506, \lambda_{2}=1.343, \lambda_{3}=-3.326, \lambda_{4}=-5.231, \lambda_{5,6}=-1.133 \pm 3.548 \mathrm{i}
$$

In simulation with the control law (24) applied to the nonlinear model (1) of the threelink biped it is possible to start with an initial configuration $\mathrm{q}(0)=\left[2.9^{\circ} ; 180^{\circ} ; 180^{\circ}\right]$. Thus, adding a joint in the haunch between the trunk and the stance leg leads to increasing the basin of attraction. The graphs of the variables $\mathrm{q}_{1}(\mathrm{t}), \mathrm{q}_{2}(\mathrm{t})$ and $\mathrm{q}_{3}(\mathrm{t})$ as function of time are shown in Figure 6 . At the end, the biped is steered to the vertical posture. The maximum torque of the actuator is applied at initial time, as shown in Figure 7. Figure 8 shows that during the stabilization process of the three-link biped the vertical component of the ground reaction is always positive and equals the weight of the biped at the end of the process.

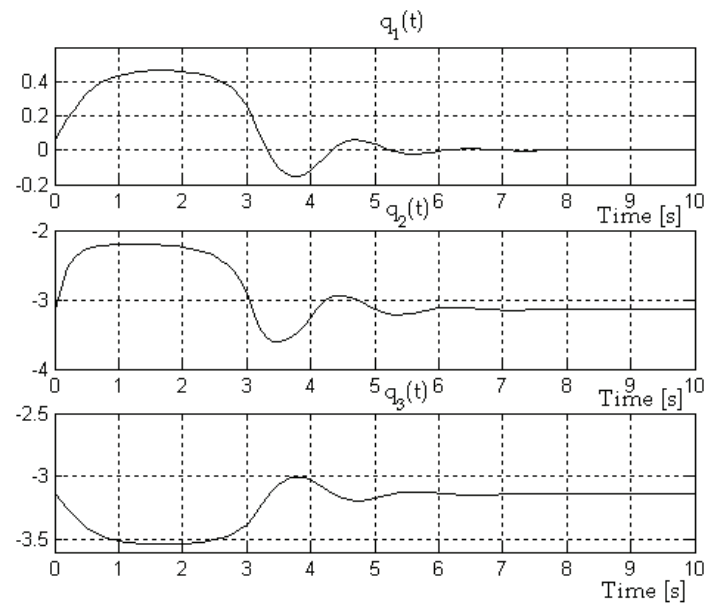

Fig. 6. Stabilization of the three-link biped in vertical posture, $q_{1}(t) \rightarrow 0, q_{2}(t) \rightarrow-\pi$ and $\mathrm{q}_{3}(\mathrm{t}) \rightarrow-\pi$ as $\mathrm{t} \rightarrow \infty$. 

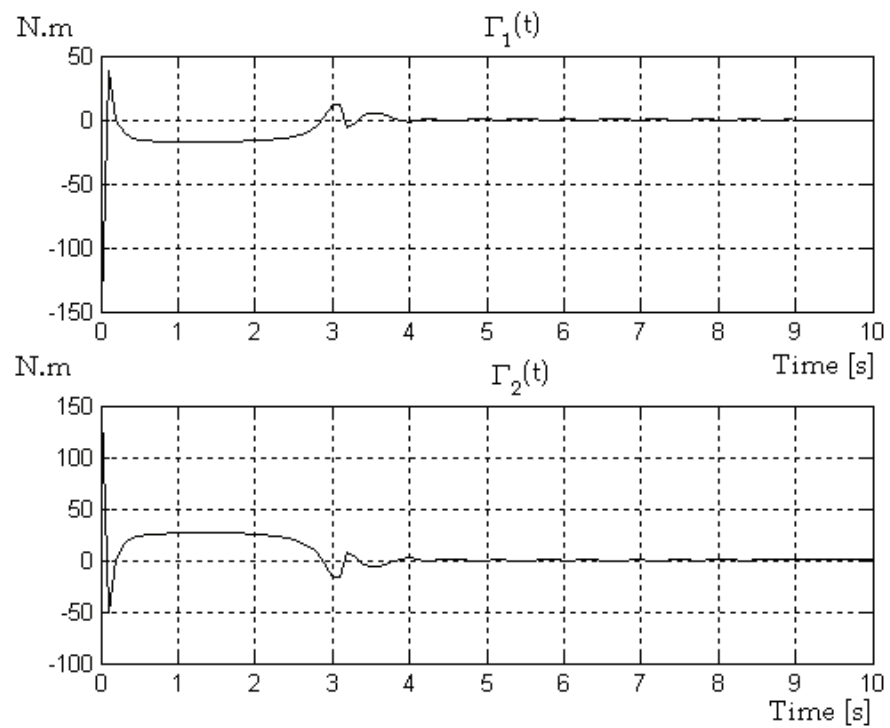

Fig. 7. Stabilization of the three-link biped in vertical posture, torques in the inter-link joints.

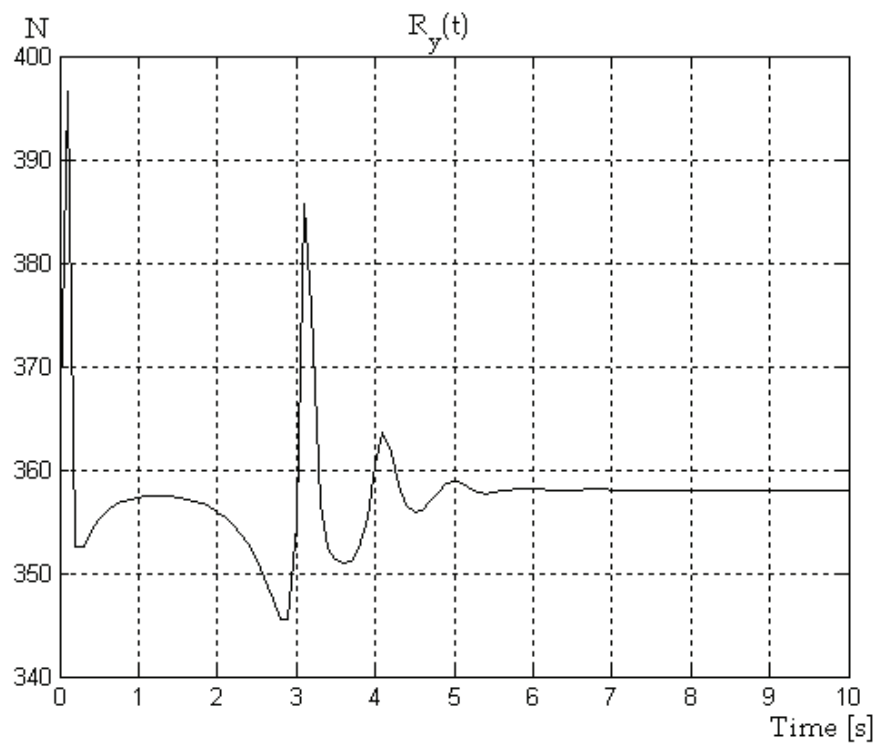

Fig. 8. Stabilization of the three-link biped in vertical posture, vertical component of the ground reaction in the stance leg tip.

\section{Five-Link Biped $(n=5)$}

In this section, we design a control law for four inter-link torques to stabilize the vertical posture of the five-link planar biped without feet in single support. 


\subsection{Control design for the five-link biped}

If the coefficient of the viscous friction $f=0$, the characteristic equation of the matrix $A$ is the following

$$
a_{0} p^{5}+a_{1} p^{4}+a_{2} p^{3}+a_{3} p^{2}+a_{4} p+a_{5}=0, p=\lambda^{2}
$$

with

$$
\begin{aligned}
& \mathrm{a}_{0}=\operatorname{det} \mathrm{D}_{1}, \mathrm{a}_{5}=\operatorname{det} \mathrm{G}_{l}= \\
& -\mathrm{g}^{5} \mathrm{~m}_{1} \mathrm{~m}_{3} \mathrm{~s}_{1} \mathrm{~s}_{3}\left(\mathrm{~m}_{1} \mathrm{l}_{2}+\mathrm{m}_{2} \mathrm{~s}_{2}\right)\left[\left(2 \mathrm{~m}_{2}+\mathrm{m}_{3}\right) \mathrm{l}_{1}+\mathrm{m}_{1}\left(2 \mathrm{l}_{1}-\mathrm{s}_{1}\right)\right]\left[\left(\mathrm{m}_{1}+\mathrm{m}_{3}\right) \mathrm{l}_{2}+\mathrm{m}_{2}\left(2 \mathrm{l}_{2}-\mathrm{s}_{2}\right)\right]
\end{aligned}
$$

According to Sylvester's theorem (Chetaev, 1962), (Chetaev, 1989) the roots $p_{1}, p_{2}, p_{3}$, $\mathrm{p}_{4}, \mathrm{p}_{5}$ the characteristic equation (25) are real values. The leading coefficient $\mathrm{a}_{0}$ of the equation (25) is positive. The free term $a_{5}$ is negative because the differences $2 l_{1}-s_{1}$ and $\mathrm{2}_{2}-\mathrm{s}_{2}$ are positive. Thus, according to Viet formula $\mathrm{p}_{1} \mathrm{p}_{2} \mathrm{p}_{3} \mathrm{p}_{4} \mathrm{p}_{5}=-\frac{\mathrm{a}_{5}}{\mathrm{a}_{0}}$, the product $\mathrm{p}_{1} \mathrm{p}_{2} \mathrm{p}_{3} \mathrm{p}_{4} \mathrm{p}_{5}$ is positive. This means that two or four roots are negative and respectively three roots or one root are positive. It is possible to prove (see (Chetaev, 1962), (Chetaev, 1989)) that the three roots, for example $p_{1}, p_{2}, p_{3}$ are positive and two roots are negative. Then the spectrum of the matrix A contains three real positive eigenvalues, three real negative eigenvalues and two pairs of pure imaginary conjugate eigenvalues. If $f \neq 0$, then spectrum of the matrix A contains three values in the right-half complex plane, and seven values in the left-half complex plane.

Let $\lambda_{i},(i=1,2,3)$ be real positive eigenvalues. Similarly to the previous cases let us consider the first three lines of the system (10) corresponding to these three positive eigenvalues,

$$
\begin{aligned}
& \dot{\mathrm{y}}_{1}=\lambda_{1} \mathrm{y}_{1}+\mathrm{d}_{11} \Gamma_{1}+\mathrm{d}_{12} \Gamma_{2}+\mathrm{d}_{13} \Gamma_{3}+\mathrm{d}_{14} \Gamma_{4} \\
& \dot{\mathrm{y}}_{2}=\lambda_{2} \mathrm{y}_{2}+\mathrm{d}_{21} \Gamma_{1}+\mathrm{d}_{22} \Gamma_{2}+\mathrm{d}_{23} \Gamma_{3}+\mathrm{d}_{24} \Gamma_{4} \\
& \dot{\mathrm{y}}_{3}=\lambda_{3} \mathrm{y}_{3}+\mathrm{d}_{31} \Gamma_{1}+\mathrm{d}_{32} \Gamma_{2}+\mathrm{d}_{33} \Gamma_{3}+\mathrm{d}_{34} \Gamma_{4}
\end{aligned}
$$

Now we want to suppress the instability of the three variables $y_{i},(i=1,2,3)$. It can be achieved by choosing the controls $\Gamma_{\mathrm{i}}(\mathrm{i}=1,2,3,4)$ such that

$$
\begin{aligned}
& \mathrm{d}_{11} \Gamma_{1}+\mathrm{d}_{12} \Gamma_{2}+\mathrm{d}_{13} \Gamma_{3}+\mathrm{d}_{14} \Gamma_{4}=\gamma_{1} \mathrm{y}_{1} \\
& \mathrm{~d}_{21} \Gamma_{1}+\mathrm{d}_{22} \Gamma_{2}+\mathrm{d}_{23} \Gamma_{3}+\mathrm{d}_{24} \Gamma_{4}=\gamma_{2} \mathrm{y}_{2} \\
& \mathrm{~d}_{31} \Gamma_{1}+\mathrm{d}_{32} \Gamma_{2}+\mathrm{d}_{33} \Gamma_{3}+\mathrm{d}_{34} \Gamma_{4}=\gamma_{3} \mathrm{y}_{3}
\end{aligned}
$$

with the three conditions,

$$
\lambda_{\mathrm{i}}+\gamma_{\mathrm{i}}<0 \quad(\mathrm{i}=1,2,3)
$$

For the system (8), (9) with $n=5$ under the control, defined by equalities (27) and inequalities (28), three positive eigenvalues $\lambda_{1}, \lambda_{2}$ and $\lambda_{3}$ of the matrix $A$ are replaced by negative values $\lambda_{1}+\gamma_{1}, \lambda_{2}+\gamma_{2}$ and $\lambda_{3}+\gamma_{3}$ respectively. All another eigenvalues do not change. 
The system (27) contains three algebraic equations with four unknown variables $\Gamma_{1}, \Gamma_{2}, \Gamma_{3}$ and $\Gamma_{4}$. Therefore, the system (27) has an infinite number of solutions. We find a unique solution of the system (27) at each step of the control process by minimizing the following functional

$$
\mathrm{J}=\max _{\mathrm{i}=1, \ldots, 4}\left|\Gamma_{\mathrm{i}}\right|
$$

This yields the torques $\Gamma_{\mathrm{i}}^{0}\left(\mathrm{y}_{1}, \mathrm{y}_{2}, \mathrm{y}_{3}\right) \quad(\mathrm{i}=1,2,3,4)$. Finally, we apply the torques $\Gamma_{\mathrm{i}}\left(\mathrm{y}_{1}, \mathrm{y}_{2}, \mathrm{y}_{3}\right),(\mathrm{i}=1,2,3,4)$, limited by the same value $\mathrm{U}$,

$$
\Gamma_{\mathrm{i}}=\Gamma_{\mathrm{i}}\left(\mathrm{y}_{1}, \mathrm{y}_{2}, \mathrm{y}_{3}\right)=\left\{\begin{array}{cll}
\mathrm{U}, & \text { if } & \Gamma_{\mathrm{i}}^{0}\left(\mathrm{y}_{1}, \mathrm{y}_{2}, \mathrm{y}_{3}\right) \geq \mathrm{U} \\
\Gamma_{\mathrm{i}}^{0}\left(\mathrm{y}_{1}, \mathrm{y}_{2}, \mathrm{y}_{3}\right), & \text { if } & \mid \Gamma_{\mathrm{i}}^{0}\left(\mathrm{y}_{1}, \mathrm{y}_{2}, \mathrm{y}_{3}\right) \leq \mathrm{U} \quad(\mathrm{i}=1,2,3,4) \\
-\mathrm{U}, & \text { if } & \Gamma_{\mathrm{i}}^{0}\left(\mathrm{y}_{1}, \mathrm{y}_{2}, \mathrm{y}_{3}\right) \leq-\mathrm{U}
\end{array} \quad\right.
$$

The torques in (30) ensure asymptotical stability of the equilibrium $y_{i}=0 \quad(i=1,2,3)$ of the first, second and third equations of the system (10) (i.e., of the system (26)), if the initial state belongs to some basin of attraction $\mathrm{V}$ in the three-dimensional space $\mathrm{y}_{1}, \mathrm{y}_{2}, \mathrm{y}_{3}$. However, the equilibrium point $y_{i}=0 \quad(i=4, \ldots, 10)$ of the fourth - tenth equations of the system (10) is also asymptotically stable for all initial conditions $\mathrm{y}_{\mathrm{i}}(0) \quad(\mathrm{i}=4, \ldots, 10)$ because $\operatorname{Re} \lambda_{\mathrm{i}}<0$ for $i=4, \ldots, 10$. Note that $y_{i} \rightarrow 0 \quad(i=1,2,3)$ as $t \rightarrow \infty$, if $\quad\left(y_{1}(0), y_{2}(0), y(0)\right) \subset V$. Consequently, according to the expressions (27), (29), (30) $\Gamma_{\mathrm{i}}(\mathrm{t}) \rightarrow 0 \quad(\mathrm{i}=1,2,3,4)$ as $\mathrm{t} \rightarrow \infty$. Thus, under the control (30) and with the conditions (28), the origin $x=0$ is an asymptotically stable equilibrium of the system (8) with some basin of attraction $V \subset Q$. The variables $y_{i}(i=1,2,3)$ depend on the original variables from vector $x$ according to the transformation $y=S^{-1} x$. Due to this, the formula (30) defines the control feedback, as a function of the vector $x$ of the original variables.

\subsection{Numerical results for the five-link biped}

The eigenvalues of the matrix $A$ for the used parameters and $f=6.0 \mathrm{~N} \cdot \mathrm{m} / \mathrm{s}$ are the following

$\lambda_{1}=5.290, \quad \lambda_{2}=3.409, \quad \lambda_{3}=1.822, \quad \lambda_{4}=-5.824, \quad \lambda_{5}=-6.355, \quad \lambda_{6}=-11.342$, $\lambda_{7,8}=-1.162 \pm 3.512 \mathrm{i}, \lambda_{9}=-0.610, \lambda_{10}=-3.303$.

In simulation with the control law (30) applied to the nonlinear model (1) of the five-link biped, it is possible to start with an initial configuration $\mathrm{q}(0)=\left[1.7^{\circ} ; 180^{\circ} ; 180^{\circ} ; 180^{\circ} ; 180^{\circ}\right]$. The angles $\mathrm{q}_{1}(\mathrm{t})$ and $\mathrm{q}_{2}(\mathrm{t}), \mathrm{q}_{3}(\mathrm{t}), \mathrm{q}_{4}(\mathrm{t}), \mathrm{q}_{5}(\mathrm{t})$ as functions of time are shown in Figures 9, 10, 11. At the end the biped is steered to the vertical posture. All the potential of the actuator is applied at initial time, as shown in Figures 12, 13.

Figure 14 shows that during the stabilization process of the five-link biped, the vertical component of the ground reaction is always positive and equals the weight of the biped at 
the end of the process.

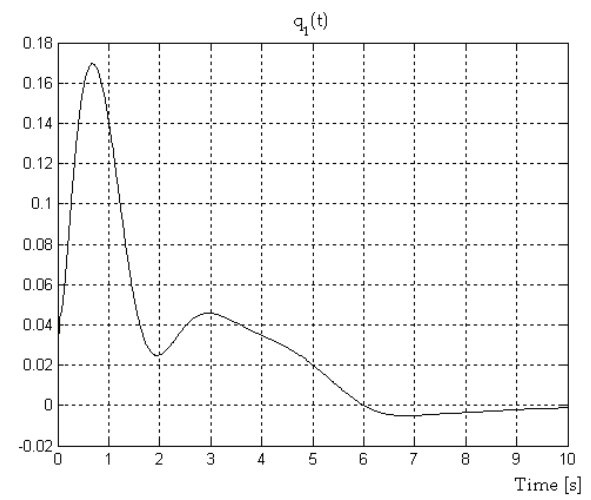

Fig. 9. Stabilization of the five-link biped in vertical posture, $q_{1}(t) \rightarrow 0$ as $t \rightarrow \infty$.
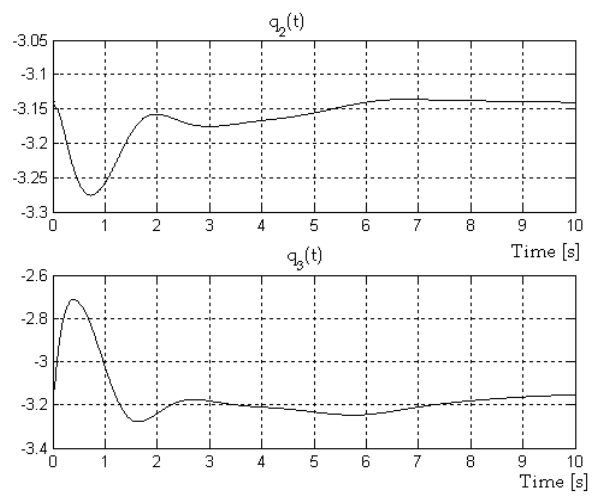

Fig. 10. Stabilization of the five-link biped in vertical posture, $\mathrm{q}_{2}(\mathrm{t}) \rightarrow-\pi, \mathrm{q}_{3}(\mathrm{t}) \rightarrow-\pi$ as $t \rightarrow \infty$.
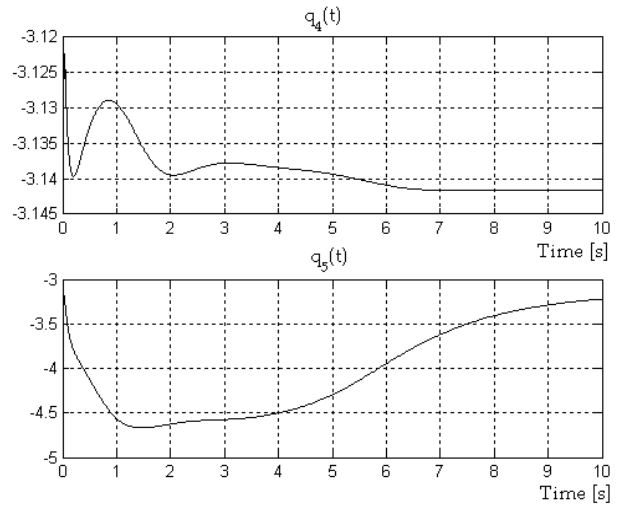

Fig. 11. Stabilization of the five-link biped in vertical posture, $\mathrm{q}_{4} \rightarrow-\pi, \mathrm{q}_{5} \rightarrow-\pi$ as $\mathrm{t} \rightarrow \infty$. 

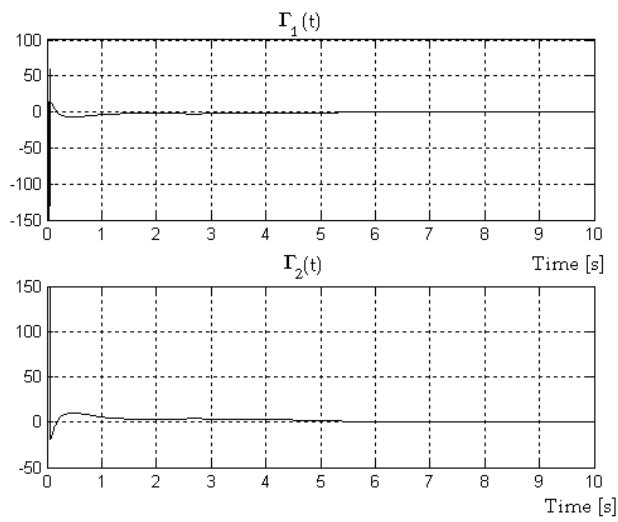

Fig. 12. Stabilization of the five-link biped in vertical posture, torques in the inter-link joints 1 and 2.
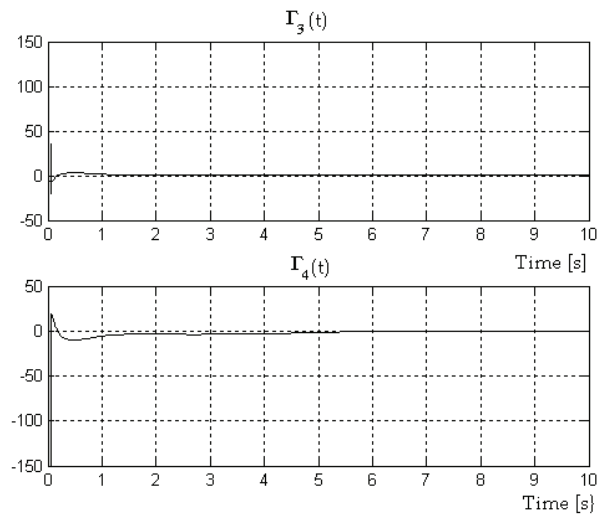

Fig. 13. Stabilization of the five-link biped in vertical posture, torques in the inter-link joints 3 and 4.

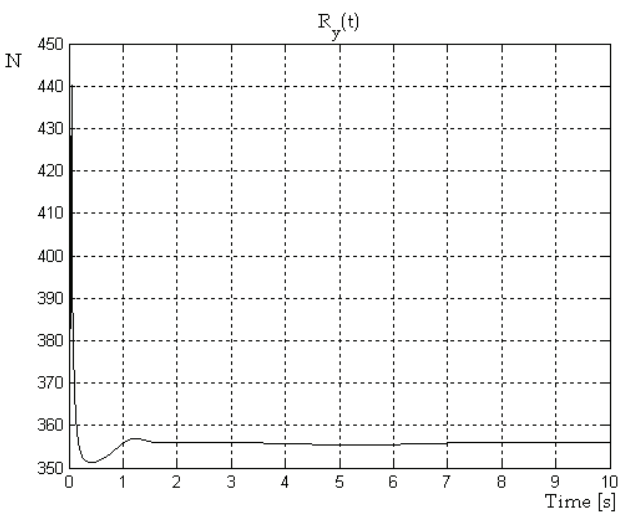

Fig. 14. Stabilization of the five-link biped in vertical posture, vertical component of the ground reaction in the stance leg tip. 


\section{Conclusion}

We defined here control strategies to stabilize the equilibrium vertical posture of a two-link, a three-link and a five-link biped, explicitly taking into account the limits imposed on the amplitudes of the control torques. For each biped we use the Jordan form of its linear model to extract the unstable modes that we want to suppress with the feedback control. For the two-link biped, the control law is optimal. This means that the basin of attraction for its linear system is as large as possible, i.e., it coincides with the controllability domain. For the five-link biped several choices of torques are allowable because we have four torques and three unstable modes. Therefore, we define a criteria to compute these torques. All the numerical results in this paper are realistic. A perspective for the case of the three-link biped is to define a control law, for which the basin of attraction is as large as allowable.

\section{References}

Aoustin, Y. \& Formal'sky, A. (2003). Control design for a biped: Reference trajectory based on driven angles as function of the undriven angle, Journal of Computer and System Sciences International, Vol. 42, No. 4, (July-August 2003) 159-176.

Aoustin, Y.; Formal'sky, A. \& Martynenko Y. (2005). A Flywheel to Stabilize a Two-Link Pendulum, Proceedings of the World Congress IFAC, Prague, Czech, July 2005 (Cdrom).

Aoustin, Y.; Formal'sky, A. \& Martynenko Y. (2006). Stabilization of unstable equilibrium postures of a two-link pendulum using a flywheel, Journal of Computer and System Sciences International, Vol. 45, No. 2, (March-April 2006) 43-50.

Beznos A. V.; Grishin, A. A.; Lenskii, A. V.; Okhotsimsky, D. E.; Formal'skii, A. M. (2003). Pendulum controled by a flywheel, Doklady Mathematics, Vol. 392, No. 6, (October 2003) 743-749.

Cambrini, L.; Chevallereau, C.; Moog, C. H. \& Stojic, R. (2001). Stable trajectory tracking for biped robots, Proceedings of IEEE Conference Decision and Control, pp. 4815-4820, Orlando Florida, December 2001.

Canudas de Wit, C.; Espiau, B. \& Urrea, C. (2002). Orbital stabilization of underactuated mechanical system, Proceedings of the World Congress IFAC, Barcelona, Spain, July 2002 (Cdrom).

Chetaev, N. G. (1962). Motion stability. Activities on an analytical mechanics, A S USSR, Moscow [In Russian].

Chetaev, N. G. (1989). Theoretical Mechanics, Springer Verlag (Mir Plublisher).

Chevallereau, C.; Abba, G.; Aoustin, Y.; Plestan, F. ; Westervelt, E. R.; Canudas de Wit, C. \& Grizzle, J. W. (2003). Rabbit: A testbed for advanced control theory, IEEE Control Systems Magazine, Vol. 23, (October 2003) No. 5, 57-78.

Chevallereau, C.; Formal'sky, A. \& Djoudi, D. (2004). Tracking of a joint path for the walking of an underactuated biped, Robotica, Vol. 22, No. 1, (January-February 2004) 15-28.

Formal'sky, A. M. (1974). Controllability and stability of systems with limited resources, Nauka, Moscow [In Russian].

Formal'skii, A. M. (2006). Stabilization of an inverted pendulum with a fixed or movable suspension point, Doklady Mathematics, Vol. 73. 406, No. 2, (January 2006) 175 - 179.

Grishin, A. A.; Lenskii, A. V.; Okhotsimsky, D. E.; Panin, D. A. \& Formal'sky, A. M. (2002). A control synthesis for an unstable object. An inverted pendulum, Journal of Computer and System Sciences International, Vol. 41, No. 5, (September-October 2002) 685-694. 
Grizzle, J.; Abba, G. \& Plestan, F. (2001). Asymptotically stable walking for biped robots: analysis via systems with impulse effects, IEEE Transaction on Automatic Control, Vol. 46, No. 1, (Junuary 2001) 51-64.

Kalman, R. E.; Falb, P. L. \& Arbib, M. A. (1969). Topics in mathematical systems theory, Mc Grow-Hill Book Compagny, New-York, San Francisco, St. Louis, Toronto, London, Sydney.

Martynenko, Yu. G. \& Formal'sky, A. M. (2005).

a). The theory of the control of a monocycle, Journal of Applied Mathematics and Mechanics, Vol. 69, No. 4, (July-August 2005) 569-583.

b). Control problems for the unstable systems, Successes in Mechanics, No. 2, (March-April 2005) 73-135, [In Russian].

Ogata, K. (1990). Modern control engineering, Prentice Hall International, Inc.

Slotine, J. J. E. \& Li. W. (1991). Applied nonlinear control, Englewood Cliffs, New Jersey: Prentice-Hall.

Spong, M. W.; Lozano, R. \& Mahony, R. (2000). An almost linear biped, Proceedings of IEEE Conference on Decision and Control, pp. 4803-4808, Sydney Australia, December 2000.

Westervelt, E. R.; Grizzle, J. W. \& Koditschek, D. E. (2003). Hybrid zero dynamics of planar biped walkers, IEEE Transactions on Automatic Control, Vol. 48, No. 1, (February 2003) 42-56. 


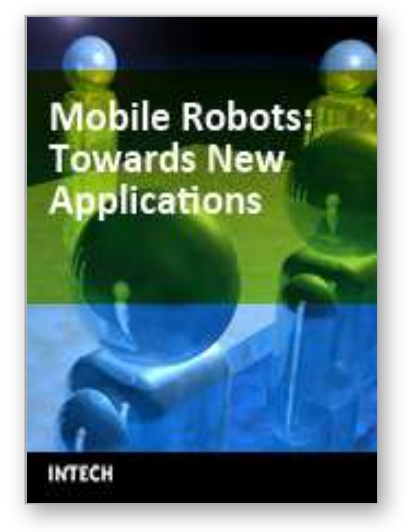

\author{
Mobile Robots: towards New Applications \\ Edited by Aleksandar Lazinica
}

ISBN 978-3-86611-314-5

Hard cover, 600 pages

Publisher I-Tech Education and Publishing

Published online 01, December, 2006

Published in print edition December, 2006

The range of potential applications for mobile robots is enormous. It includes agricultural robotics applications, routine material transport in factories, warehouses, office buildings and hospitals, indoor and outdoor security patrols, inventory verification, hazardous material handling, hazardous site cleanup, underwater applications, and numerous military applications. This book is the result of inspirations and contributions from many researchers worldwide. It presents a collection of wide range research results of robotics scientific community. Various aspects of current research in new robotics research areas and disciplines are explored and discussed. It is divided in three main parts covering different research areas: Humanoid Robots, Human-Robot Interaction, and Special Applications. We hope that you will find a lot of useful information in this book, which will help you in performing your research or fire your interests to start performing research in some of the cutting edge research fields mentioned in the book.

\title{
How to reference
}

In order to correctly reference this scholarly work, feel free to copy and paste the following:

Formalsky Alexander and Aoustin Yannick (2006). Biped without Feet in Single Support: Stabilization of the Vertical Posture with Internal Torques, Mobile Robots: towards New Applications, Aleksandar Lazinica (Ed.), ISBN: 978-3-86611-314-5, InTech, Available from:

http://www.intechopen.com/books/mobile_robots_towards_new_applications/biped_without_feet_in_single_su pport_stabilization_of_the_vertical_posture_with_internal_torques

\section{INTECH}

open science | open minds

\section{InTech Europe}

University Campus STeP Ri

Slavka Krautzeka 83/A

51000 Rijeka, Croatia

Phone: +385 (51) 770447

Fax: +385 (51) 686166

www.intechopen.com

\section{InTech China}

Unit 405, Office Block, Hotel Equatorial Shanghai

No.65, Yan An Road (West), Shanghai, 200040, China

中国上海市延安西路65号上海国际贵都大饭店办公楼405单元

Phone: +86-21-62489820

Fax: $+86-21-62489821$ 
(C) 2006 The Author(s). Licensee IntechOpen. This chapter is distributed under the terms of the Creative Commons Attribution-NonCommercialShareAlike-3.0 License, which permits use, distribution and reproduction for non-commercial purposes, provided the original is properly cited and derivative works building on this content are distributed under the same license. 